\title{
Placental type alkaline phosphatase tissue expression in ovarian serous carcinoma
}

\author{
Maria Orsaria ${ }^{\mathrm{a}, *}$, Ambrogio P. Londero $^{\mathrm{b}, \mathrm{c}, *}$, Stefania Marzinotto $^{\mathrm{a}}$, Carla Di Loreto ${ }^{\mathrm{a}}$, \\ Diego Marchesonic and Laura Mariuzzi ${ }^{\mathrm{a}}$ \\ ${ }^{a}$ Department of Medical and Biological Sciences, DSMB, University of Udine, Udine, Italy \\ ${ }^{\mathrm{b}}$ Unit of Obstetrics and Gynecology, Hospital of Monfalcone (GO), Italy \\ ${ }^{\mathrm{c}}$ Department of Experimental Clinical and Medical Science, DISM, Clinic of Obstetrics and Gynecology, \\ University of Udine, Udine, Italy
}

\begin{abstract}
.
OBJECTIVE: To analyze the expression profile of placental type alkaline phosphatase (PLAP), cancer antigen 125 (CA125), and human epididymis protein 4 (HE4) in serous ovarian cancer and to correlate their expression with the tumor aggressiveness and progression.

METHODS: Retrospective study considering a tissue microarray of 82 women affected by ovarian serous cancer. Protein expression was assessed by immunohistochemistry on ovarian serous cancer tissue samples. Immunohistochemical staining was semiquantitatively evaluated as $\mathrm{H}$-score.

RESULTS: Median H-score values were lower for PLAP, 1 (IQR 0-4) than CA125, 10 (IQR 6-12) or HE4, 8 (IQR 5-12). Even if PLAP was less expressed in the cells of serous ovarian cancer than CA125 or HE4 it was relatively more expressed in the fourth quartile of its H-score distribution among cases with low CA125 or HE4 expression. Furthermore, PLAP and HE4 high expression resulted to be significantly correlated with a better prognosis.

CONCLUSIONS: PLAP could be an additional marker for early detection of serous ovarian carcinoma, together with the established CA125 and HE4. In addition, PLAP expression is correlated with prognosis, giving, in this way, an additional tool for improving treatment approach.
\end{abstract}

Keywords: PLAP, HE4, CA125, survival, prognostic factors, ovarian serous cancer

\section{Introduction}

Ovarian carcinoma is still nowadays a lethal disease and its incidence has remained almost stable over the decades. This is due mostly to the delays in diagnosis because this type of cancer produces few local symptoms and presents late in most cases, with fewer than $25 \%$ of cases detected in stage I (International Federation of Gynecology and Obstetrics staging) [4,11]. When ovarian cancer is diagnosed in stage I, up to $90 \%$ of patients can be cured [4].

\footnotetext{
* Corresponding author: Ambrogio P. Londero, Unit of Obstetrics and Gynecology, Hospital of Monfalcone, via Galvani 1, 34074 Monfalcone (GO), Italy. Tel.: +39 0432 559635; Fax: +39 0432 559641; E-mail: ambrogio.londero@ gmail.com.
}

A lot of efforts in the past years were made to establish effective screening strategies to improve clinical outcome, and in this way survival rates have improved steadily, thanks to the advances in surgical management, development of effective cytotoxic drugs and the route of administration of chemotherapy [17].

At the present moment, screening methods include transvaginal ultrasound, serum markers and the combination of both [20]. Among the serum markers, one of the most well characterized and used is cancer antigen 125 (CA125), that, if serially determined in blood samples can achieve a good specificity (up to $99.6 \%$ ) [4], but its sensitivity and specificity are limited by the facts that many benign gynecological and medical conditions, as well as other malignancies can elevate CA125 levels and $20 \%$ of stage I ovarian can-

ISSN 1574-0153/16/\$35.00 (c) 2016 - IOS Press and the authors. All rights reserved

This article is published online with Open Access and distributed under the terms of the Creative Commons Attribution Non-Commercial License (CC BY-NC 4.0). 
cers express little or no CA125 [17]. Another interesting marker that emerged recently is human epididymis protein 4 (HE4) [17] that resulted to be over-expressed by epithelial ovarian cancer tumors and circulate in the serum of patients with this disease [7]. HE4 has the same sensitivity as CA125 for detecting malignancy, however it is expressed in a subset of ovarian cancers that do not express CA125, suggesting that could be better the combination of both markers [19]. Nevertheless, even in combination serum biomarkers have limited sensitivities and specificities, so these limitations have inspired the evaluation of novel tumor markers to improve the ability to predict malignancy in women with pelvic masses.

More than 20 years ago, there has been considerable interest in the study of placental-like alkaline phosphatase (PLAP) as a marker of epithelial ovarian cancer [8]. PLAP is normally produced by the syncytiotrophoblast of the placenta and it is normally undetectable in blood of healthy subjects [8]; human PLAP was one of the first oncofetal antigens found to be ectopically expressed by tumor cells and this antigen is a useful marker expressed in several malignancies including choriocarcinoma, seminoma, and ovarian carcinoma [21], but also in gastrointestinal tract tumors [10]. Many studies in the past evaluated the immunohistochemical expression or the presence in the serum of PLAP antigen in relation to tumor features, with different and discordant results; Kellen et al. found that, among the gynecological tumors, only in cancers of the ovaries, there was a positive correlation between this enzyme marker and the extent of the disease (tumor burden) [13]; Stigbrand et al. reported that PLAP is positively correlated to tumor burden and the outcome of the disease [24]; Tholander et al. showed that PLAP levels were significantly influenced by histological type of tumor and by grade of differentiation but not by clinical stage [25].

In this study, PLAP, CA125, and HE4 were evaluated by immunohistochemistry on 82 patients with a diagnosis of serous ovarian carcinoma. We also correlate the expression of PLAP, CA125, and HE4 in the neoplastic cell with the survival of the patients to offer a new potential prognostic marker.

\section{Materials and methods}

\subsection{Patients and specimens}

The majority of the present cases were used for a previous study about high grade serous ovarian cancer [14]. As previously described, from the registry of the Pathology Department of the University Hospital of Udine, a total of 105 patients who had a pathological diagnosis of ovarian serous carcinoma between January 2003 and July 2008 were identified. We included only primary interventions with a diagnosis of ovarian serous carcinoma and we excluded all patients who performed chemotherapy before surgery. Therefore, a total of 82 patients were included in this study. The excluded cases were 20 patients with intraperitoneal recurrences and 3 patients who performed neoadjuvant therapy after laparoscopic staging but before primary surgery. All these cases were staged according to the International Federation of Gynecology and Obstetrics (FIGO) staging system [11] (Stages I, II, III, IV) and to the TNM classification of malignant tumors for ovarian cancer [9]; the histopathological grading of these cases was evaluated using Malpica System [15] (low- and high-grade). Other information such as clinical history and management for each patient were gathered using clinical files. Data included age at intervention, date and radicality of intervention, contingent assumption of any hormonal or non-hormonal therapy, parity, gravidity, last menses before intervention, familial history of cancer (ovary or breast), and clinical follow up until January 2011. In archived clinical records, follow up consisted of data collected during medical examination (unexplained weight loss, presence of ascites), diagnostic imaging (ultrasound, CT, or PET-FDG/CT), blood tests (increase of serum CA125), and second-look surgery. We recorded in detail also first line chemotherapy and during the study period patients were treated with either single agent chemotherapy (carboplatin) or platinum-based combination chemotherapy (carboplatin and paclitaxel). Patients with FIGO stage IA or IB with low grade were not treated with adjuvant chemotherapy (NCCN guidelines, last access May 2012). Platinum resistance was defined as disease progression during first line chemotherapy or relapse within 6 months after treatment $[2,14]$.

\subsection{Preparation of tissue microarray slides}

Tissue microarray (TMA) technique is able to simultaneously analyze multiple formalin fixed paraffinembedded tissues. Haematoxylin-Eosin ( $\mathrm{H}$ and $\mathrm{E})$ stained sections were carefully examined to select representative regions from each selected donor block. 
TMAs were made using a Beecher tissue micro-arrayer and a cylindrical, thin-walled needle (inner diameter of $1.5 \mathrm{~mm}$ ) was used for coring tissues and for transferring cored samples into array cavities in the recipient block. A total of 82 blocks were available and for each block we performed two core biopsies, representative of the primary carcinoma (which involved one or both ovaries) and the metastasis, where specimen was available. From the recipient block, we obtained $4-\mu$ m-thick transverse sections.

For antigen retrieval and deparaffinization, slides were heated for 20 minutes at $98^{\circ} \mathrm{C}$ in Target Retrieval Solution according to manufacturer's instructions (Dako K8005, Glostrup, DK) with PT-link (Dako). Then the slides were incubated at room temperature in $\mathrm{H} 2 \mathrm{O} 2$ for 10 minutes to block endogenous peroxidase activity. The sections were rinsed in PBS and then incubated in a wet chamber at room temperature for 1 hour with the following primary antibodies: monoclonal PLAP (PLAP mouse DAKO M7191, diluted 1:200), CA125 (CA125 mouse DAKO M3520, diluted 1:20), and HE4 (HE4 - rabbit ABCAM ab24480, diluted 1:150). A Dako REAL ${ }^{\mathrm{TM}}$ EnVision ${ }^{\mathrm{TM}}$ Dako Rabbit/Mouse (Dako, K5007, Glostrup, DK) was used as second antibody. HRP activity was detected using Dako REAL ${ }^{\mathrm{TM}}$ DAB + Chromogen (Dako, K5007, Glostrup, DK) as substrate for $3 \mathrm{~min}$ in accordance with the manufacturer's instructions. Sections were counterstained with hematoxylin with cover slip. Each marker was optimized using as positive controls various normal tissue samples and sections from serous ovarian cancer. In addition, sections incubated with non-immune rabbit serum instead of primary antibody were used as negative controls.

Semiquantitative analysis of the immunohistochemical staining was performed independently by two pathologists and the staining was semiquantitatively evaluated as $\mathrm{H}$-score (product of actual percentage of positive-stained cells and intensity score-evaluated as strong 3, moderate 2 and weak 1-giving a possible range of 0-300). The intraclass correlation coefficient (ICC) agreement between the two independent pathologists was respectively in case of PLAP, CA125, and HE4 0.97 (95\% C.I. 0.94-0.98), 0.98 (95\% C.I. 0.970.99 ), and 0.97 (95\% C.I. 0.95-0.98). In addition, in case of discrepancies the two pathologists together reviewed the specimen and gave a final shared score. In case of disagreement they reviewed the case with a third pathologist.
Table 1

Description of our population

\begin{tabular}{ll} 
Age at intervention (years) & $63.35( \pm 12.12)$ \\
Post-menopausal status & $75.6 \%(62 / 82)$ \\
Family history of cancer & $14.6 \%(12 / 82)$ \\
Adjuvant chemotherapy & $85.4 \%(70 / 82)$ \\
Median survival after intervention (months) & $40(22-56)$ \\
Death from cancer & $54.9 \%(45 / 82)$ \\
FIGO staging & \\
I & $24.4 \%(20 / 82)$ \\
II & $15.9 \%(13 / 82)$ \\
III-IV & $59.8 \%(49 / 82)$ \\
Malpica's grading system & \\
Low grade & $11 \%(9 / 82)$ \\
High grade & $89 \%(73 / 82)$ \\
Cytoreduction among stage FIGO III & \\
Complete & $17 \%(8 / 47)$ \\
Incomplete & $83 \%(39 / 47)$ \\
First line chemotherapy & \\
Carboplatin monotherapy & \\
Carboplatin combination therapy & $11 \%(9 / 82)$ \\
No chemotherapy & $74.4 \%(61 / 82)$ \\
$\quad$ & $14.6 \%(12 / 82)$ \\
\hline
\end{tabular}

\subsection{Statistical analysis}

Data were analyzed using $R$ (version 3.1.0) and $p<$ 0.05 was considered significant. For all proteins analyzed the staining was investigated in terms of percentage of positive cells and $\mathrm{H}$-score. Normality of variables was tested with the Kolmogorov-Smirnov test. Concordance of the two pathologists was evaluated by intraclass correlation coefficient (ICC) analysis. Nonparametric data were presented with the median value and the interquartile range (IQR), whereas parametric data were described with the mean value and its standard deviation. The Kruskal-Wallis test and the one way ANOVA for continuous variables were used for the variance analysis where appropriate. For comparison of multiple categorical variables the chi-square test was used. For bivariate analysis, the following statistical tests were applied: the Wilcoxon test, t-test, Spearman's rho, and Kendall's tau for continuous variables and the Chi-square and Fisher exact test for categorical variables. Overall survival was analyzed with KaplanMeier curves and log-rank test for comparisons was performed. In this survival analysis with Kaplan-Meier curves we divided our population in two groups one group expressing the studied proteins at a level higher than the third quartile value of the distribution and the other group in which the expression was lower. Furthermore, we performed Cox proportional hazards mono- and multivariate regression analysis. Predictive accuracy of Cox models was assessed by estimator of cumulative/dynamic area under the curve (AUC) proposed by Hung and Chiang [12]. 


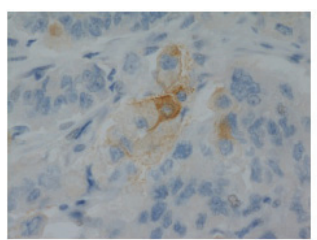

(a) PLAP, median H-score

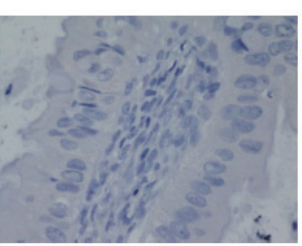

(f) $C A 125$, first quartile.

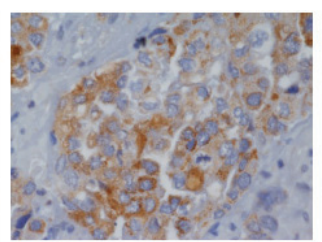

(b) HE4, median H-score.

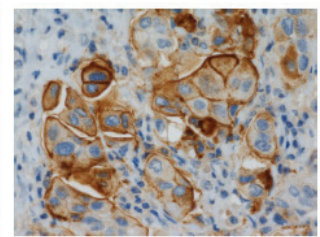

(g) PLAP, Case

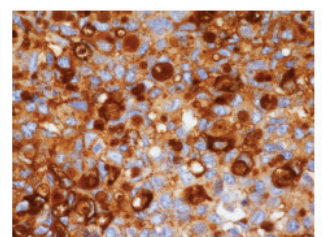

(c) CA125, median H-score

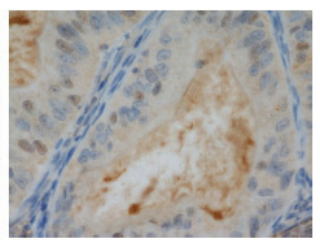

(d) PLAP, Case 1, score value in fourth quartile.

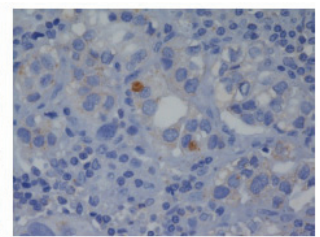

(h) HE4, quartile.

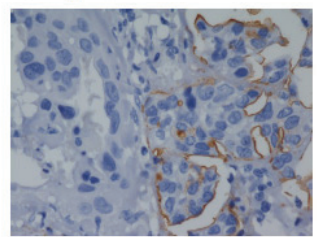

(i) CA125, Case 2, score value in second quartile.

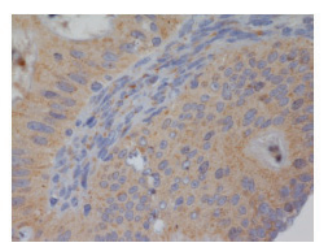

(e) HE4, Case 1, score value in fourth quartile.

Fig. 1. Placental type alkaline phosphatase (PLAP), cancer antigen 125 (CA125), and human epididymis protein 4 (HE4) expression in ovarian serous cancer by immunihistochemistry. (a): Example of median H-score value of PLAP expression (original magnification $\times 400$ ). (b): Example of median H-score value of HE4 expression (original magnification $\times 400$ ). (c): Example of median H-score value of CA125 expression (original magnification $\times 400)$. (d, e, and f): Example of PLAP and HE4 high expression and CA125 low expression in a case of low Malpica's grade ovarian serous cancer (original magnification $\times 400$ ). ( $\mathrm{g}, \mathrm{h}$, and I) Example of PLAP high expression and HE4 and CA125 low expression in a case of high Malpica's grade ovarian serous cancer (original magnification $\times 400$ ).

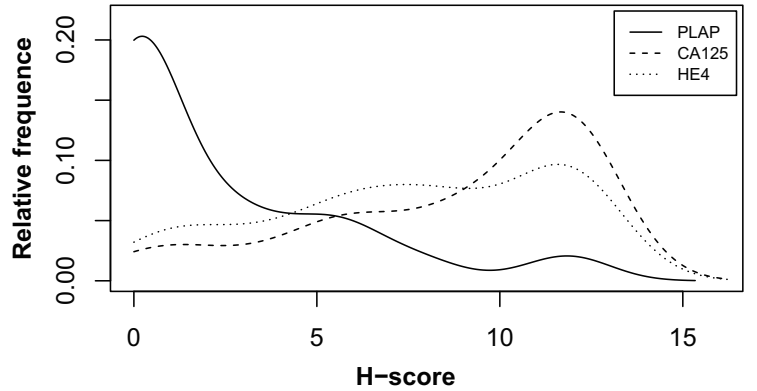

A

PLAP low expression $(71.9 \% ; 59 / 82)$

\begin{tabular}{|l|c|c|}
\hline & HE4 low expression & HE4 high expression \\
\hline CA125 low expression & $35.4 \%(29 / 82)$ & $8.5 \%(7 / 82)$ \\
\hline CA125 high expression & $15.8 \%(13 / 82)$ & $12.2 \%(10 / 82)$ \\
\hline
\end{tabular}

PLAP high expression $(28.0 \% ; 23 / 82)$

\begin{tabular}{|l|c|c|}
\hline & HE4 low expression & HE4 high expression \\
\hline CA125 low expression & $12.2 \%(10 / 82)$ & $2.4 \%(2 / 82)$ \\
\hline CA125 high expression & $7.3 \%(6 / 82)$ & $6.1 \%(5 / 82)$ \\
\hline
\end{tabular}

$\mathrm{B}$

Fig. 2. (A) Plot of H-index markers distribution. (B) Contingency table of $\mathrm{H}$-index score distribution. High marker expression is intended as the fourth quartile of $\mathrm{H}$-index score distribution while low marker expression as first-second-third quartile of $\mathrm{H}$-index score distribution.

\section{Results}

Mean age at diagnosis was 63.35 years $( \pm 12.12)$ and $75.6 \%$ of women were in post-menopause. The majority of cancers were FIGO stage III-IV and Malpica's high grade (Table 1). Median H-score values were lower for PLAP 1 (IQR 0-4) than CA125 10 (IQR 6-12) or HE4 8 (IQR 5-12) (Figs 1A-C, and Fig. 2A that shows H-score distributions). PLAP and CA125 present a localization to the cell membrane, and to the cytoplasm, while HE4 had a cytoplasmic localization. No significant differences in PLAP, HE4, or CA125 expression were observed between primary tumor and synchronous peritoneal metastases where present or between high and low Malpica's grade. Furthermore, two cases were non-expressing HE4 and PLAP but were expressing CA125. In addition, three cases were not expressing CA125 but were expressing HE4 and in one case also PLAP. We further analyzed the immunohistochemical co-expression of PLAP, CA125, and HE4 in ovarian serous cancer tissue considering the $\mathrm{H}$-score value distribution of the three markers subdivided in quartiles. In Fig. 2B we considered as high marker expression the fourth quartile of the $\mathrm{H}$-score distribution and we show that in $12.2 \%(10 / 82)$ of considered cases the PLAP is relatively high expressed while CA125 and HE4 are low expressed. In addition, we further focused on the first quartile of the distribution of HE4 and CA125 because this group is the less expressing the two considered markers. In our data we observed that in the cases with low tissue expression of CA125 or HE4, PLAP was expressing in the third or fourth quartile of its distribution (high expression) in the $43.8 \%$ of cases (7/16) of low levels of CA125 


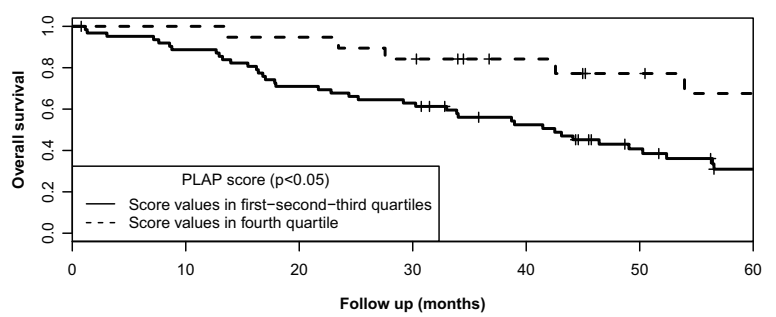

A

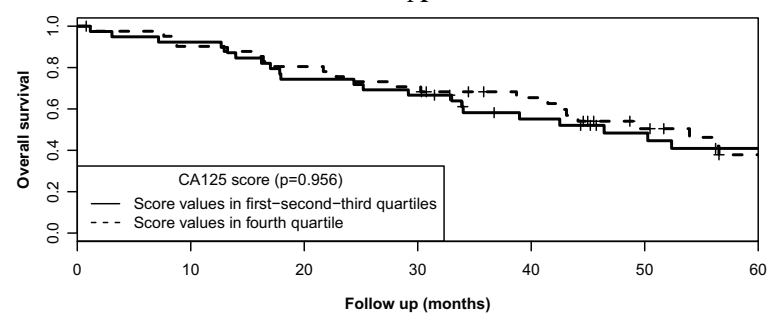

B

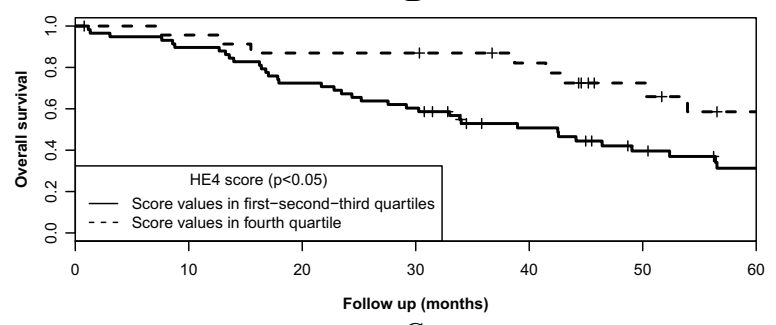

$\mathrm{C}$

Fig. 3. Kaplan-Meier curves of overall survival and studied markers immunohistochemical expression in ovarian serous cancer ( $p$-values refer to log-rank test). (A): PLAP H-score value distribution subdivided in two groups ((1) first-second-third quartiles and (2) fourth quartile of the distribution). Low immunohistochemical tissue expression of PLAP showed a shortened survival period $(p<0.05)$. (B): CA125 H-score value distribution subdivided in two groups ((1) first-second-third quartiles and (2) fourth quartile of the distribution). (C): HE4 H-score value distribution subdivided in two groups ((1) first-second-third quartiles and (2) fourth quartile of the distribution). Low immunohistochemical tissue expression of HE4 showed a shortened survival period $(p<0.05)$.

and in the $45 \%$ of cases $(9 / 20)$ of low levels of HE4 (Figs 1D-I).

No significant differences were observed in FIGO stage, Malpica's grading, treatment modalities, patients characteristics and platinum resistance between patients with high and low PLAP expression. We have only found significant lower expression of PLAP in patients with a familial history for cancer.

We analyzed also the influence of PLAP, CA125, and HE4 immunohistochemical expression on survival. In Fig. 3 we show low tissue expression of PLAP and HE4 to be associated to a significant decrease in overall survival $(p<0.05)$. In Table 2 considering the multivariate analysis only PLAP tissue expression resulted to be a significant factor influencing survival.

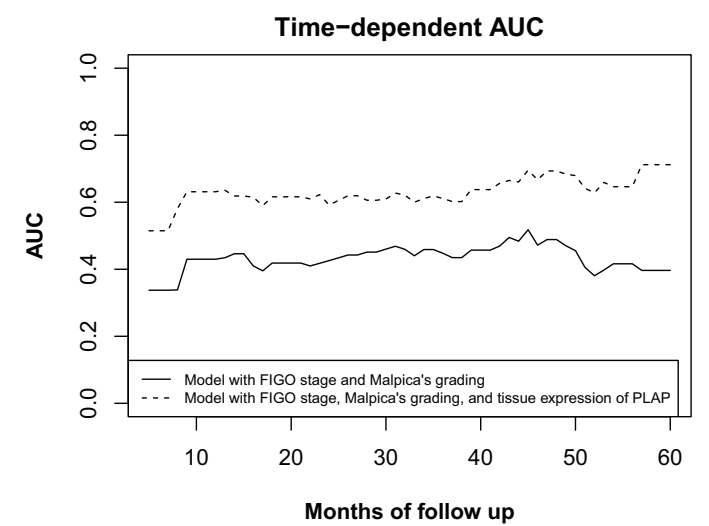

Fig. 4. Time dependent AUC values of the two Cox multivariate models.

This difference was still significant despite the correction in multivariate analysis for: age at intervention, FIGO stage, Malpica's grading, complete cytoreduction, and platinum resistance. Furthermore, considering the Cox model to predict overall survival with FIGO stage and Malpica's grading the summary measure of the time dependent AUC was 0.43 , while considering in the model also the tissue expression of PLAP the AUC was 0.63 and the difference between the two models was statistically significant $(p<0.05)$. In fact, the multivariate Cox model with FIGO stage, Malpica's grading, and tissue expression of PLAP was more predictive for survival than the model with only FIGO stage and Malpica's grading. In Fig. 4 the time dependent AUC values of the two models are shown.

\section{Discussion}

We found that even if PLAP have lower median $\mathrm{H}$-score values in serous ovarian cancer than CA125 or HE4 was commonly expressed in the fourth quartile of its $\mathrm{H}$-score distribution among cases with low CA125 or HE4 expression. Furthermore, its high expression resulted to be significantly correlated with a better prognosis.

Numerous investigators in the past have studied many possible markers in the diagnostic pathway of ovarian carcinoma, in order to facilitate earlier diagnosis and disease monitoring; some of them have been measured in the serum alone and in combination in early stage epithelial ovarian cancer: CA125, C19-9, CEA, PLAP [5] and more recently HE4. Since the publication of the initial report by Bast et al. [3] CA125 has become the accepted test with which other markers are compared; more recently HE4 [7], another effective 
Table 2

Cox proportional hazards regression models (univariate and multivariate analysis §) in serous oavrian cancers. In this table are reported hazard ratio (HR), the relative $95 \%$ confidence interval and the p-value. In the multivariate analysis ( $\$$ ) we performed a correction for the following factors: age at intervention, FIGO stage, Malpiga'a grading, complete cytoreduction, and platinum resistance

\begin{tabular}{|c|c|c|c|c|}
\hline & $\mathrm{HR}(95 \% \mathrm{CI})$ & $p$ & $\operatorname{HR}(95 \% \mathrm{CI})(\S)$ & $p(\S)$ \\
\hline PLAP score in fourth quartile of the distribution & $0.32(0.13-0.81)$ & $<0.05$ & $0.34(0.13-0.92)$ & $<0.05$ \\
\hline CA125 score in fourth quartile of the distribution & $0.77(0.42-1.42)$ & 0.401 & $1.27(0.62-2.61)$ & 0.518 \\
\hline HE4 score in fourth quartile of the distribution & $0.47(0.22-0.97)$ & $<0.05$ & $0.55(0.25-1.23)$ & 0.144 \\
\hline
\end{tabular}

marker, is recommended in combination with CA125 for early diagnosis, monitoring disease status and the efficacy of treatment [17].

Only few studies were done examining the expression of ovarian carcinoma antigens, by immunohistochemistry assays, directly on the neoplastic tissue and this is the first report to analyze the co-expression of PLAP with HE4 and CA125. In the past PLAP was considered a promising marker for epithelial ovarian cancer screening [8]. But its accuracy was limited because PLAP blood levels are strongly influenced by tobacco smoke [26]. Our data suggested a possible role of this marker in modern algorithms proposed for ovarian cancer screening in association with HE4 and CA125 to possibly improve screening accuracy [28]. In fact, in tumors with low expression of HE4 and CA125 we observed an increased expression of PLAP which may reflect increased blood levels of PLAP when HE4 and CA125 may not be significant for the screening algorithm. For these reasons it is important to plan studies that test in the same algorithm the blood levels of all these three proteins.

In previous studies, the presence of PLAP was mainly evaluated in the serum of the patients and correlated to histological type of tumor, grade of differentiation, clinical stage and outcome of the disease [13, $24,25]$. Not many studies have examined PLAP expression directly in the tissue $[10,16,18,23]$ and described different distribution of PLAP expression in serous and mucinous tumors, with diffuse cytoplasmic staining in the more malignant tumors and more frequent negativity in dedifferentiated ones, suggesting that it's possible that tumor cells express PLAP only at certain stages in the cell cycle; in this study they also reported a discrepancy between tumor tissue and circulating PLAP levels, maybe because cell membrane damage or cell death may be required before significant PLAP is released to the circulation. Stendhal et al. [23] described the expression of PLAP in $51 \%$ of the ovarian epithelial tumors examined, with similar incidence in the four major groups of the adenocarcinomas, i.e., serous, mucinous, endometrioid and mesonephric tumours.

We observed that there was a highly positive correlation between low tissue expression of PLAP or
HE4 and short survival, with a follow up of minimum 29 months. Our data confirmed previous studies that found PLAP and HE4 to correlate with cancer prognosis $[22,24]$. In addition, PLAP expression didn't show any correlation with Malpica's grade and clinical stage, results that are discordant compared to previous studies $[13,24,25]$. This observation of an inverse correlation between neoplastic cell antigen expression and survival is very interesting and point out some important tools for a different approach to the disease. It may be useful for the future to start to evaluate PLAP and other markers (eg. human apurinic endonuclease/redox factor 1-NPM1) immunohistochemical expression directly on the specimen at the time of diagnosis, to have a novel important additional pathological information about prognosis, other than the traditional indicators of prognosis i.e. grade of differentiation and clinical stage $[6,14]$. This can also potentially help in individualizing treatment for patients. For example in early stage disease (stage Ia/b), where the patients already have better prognosis, adjuvant chemotherapy may be confined only to the cases with adverse prognostic features [27]; therefore in this context as prognostic factor it would be useful in deciding which patients should receive chemotherapy in order to minimize an otherwise not justified toxicity. Moreover, this finding, as prognostic factor, can help identify subgroups of patients with especially poor prognosis and give us the advise to try to develop alternative treatment strategies for these patients [1].

The main strength of this study is the analysis of the co-expression in the same tissue samples of CA125, HE4, and PLAP. The second strength of this study is the correlation between low PLAP or HE4 H-scores and shortened survival periods. These data presented herein could influence the clinical management (new screening algorithms, prognosis determination) and planning of future therapies. Other strengths of our study are a relatively long follow up period, and the statistical power gained by analyzing a large number of cases. As previously described this TMA model was retrospectively designed [14]. This limited us in accurately assessing disease free survival and adju- 
vant treatment effectiveness that accounted for tumor chemoresistance emergence [14]. The absence of information about tobacco smoke habits in the retrospective files and the variable expression level of PLAP were limitations of the present study. Finally, other limitations of the present study are the lack of blood samples, to test the presence of the markers, and analysis of tissue from the only serous carcinomas of the ovary that although the majority are not the only malignant disease of the ovary.

\section{Conclusion}

Serous ovarian carcinoma is the leading cause of cancer death among gynecological malignancies. Despite the efforts done for the early diagnosis and the radical treatment, these goals are far to be achieved. In this setting we tried to examine a new possible marker, PLAP.

In this study we observed many different aspects in which PLAP detection could be useful in this disease. First of all, PLAP can be an additional marker for early detection of serous ovarian carcinoma, together with the established CA125 and HE4.

Secondary, PLAP expression is correlated with prognosis, giving, in this way, an additional tool for the categorization of the patients affected from ovarian serous carcinoma. Moreover, this information lead us to suppose that PLAP can be useful to address a more correct treatment approach, more aggressive in patients with low expression of PLAP and with poor prognosis.

\section{Conflict of interest}

The authors declare that they have no potential conflicts of interest relevant to this article.

\section{Acknowledgements}

We are grateful to Matteo De Luca and Federico Todaro for the technical assistance.

\section{References}

[1] Agarwal R, Kaye SB. Prognostic factors in ovarian cancer: how close are we to a complete picture? Ann Oncol 2005; 16 : 4-6.

[2] Al-Attar A, Gossage L, Fareed KR, et al. Human apurinic/ apyrimidinic endonuclease (APE1) is a prognostic factor in ovarian, gastro-oesophageal and pancreatico-biliary cancers. Br J Cancer 2010; 102: 704-709.
[3] Bast R Jr, Klug TL, St John E, et al. A radioimmunoassay using a monoclonal antibody to monitor the course of epithelial ovarian cancer. N Engl J Med 1983; 309: 883-887.

[4] Bast RC Jr. Status of tumor markers in ovarian cancer screening. J Clin Oncol 2003; 21: 200s-205s.

[5] Berek JS, Bast R Jr. Ovarian cancer screening. The use of serial complementary tumor markers to improve sensitivity and specificity for early detection. Cancer 1995; 76: 20922096.

[6] Berman ML. Future directions in the surgical management of ovarian cancer. Gynecol Oncol 2003; 90: S33-S39.

[7] Drapkin R, von Horsten HH, Lin Y, et al. Human epididymis protein 4 (HE4) is a secreted glycoprotein that is overexpressed by serous and endometrioid ovarian carcinomas. Cancer Res 2005; 65: 2162-2169.

[8] Fisken J, Leonard RC, Shaw G, et al. Serum placental-like alkaline phosphatase (PLAP): a novel combined enzyme linked immunoassay for monitoring ovarian cancer. J Clin Pathol 1989; 42: 40-45.

[9] Gospodarowicz M, Wittekind C, Sobin L, et al. TNM Classification of Malignant Tumours, 7th Edition. Wiley-Blackwell 2009 .

[10] Hamilton-Dutoit SJ, Lou H, Pallesen G. The expression of placental alkaline phosphatase (PLAP) and PLAP-like enzymes in normal and neoplastic human tissues. An immunohistological survey using monoclonal antibodies. APMIS 1990; 98: 797-811.

[11] Heintz APM, Odicino F, Maisonneuve P, et al. Carcinoma of the ovary. FIGO 26th Annual Report on the Results of Treatment in Gynecological Cancer. Int J Gynaecol Obstet 2006; 95(1): S161-S192.

[12] Hung H, Chiang CT. Estimation methods for time-dependent AUC models with survival data. Canadian Journal of Statistics 2010; 38: 8-26.

[13] Kellen JA, Bush RS, Malkin A. Placenta-like alkaline phosphatase in gynecological cancers. Cancer Res 1976; 36: 269271.

[14] Londero AP, Orsaria M, Tell G, et al. Expression and prognostic significance of APE1/Ref-1 and NPM1 proteins in high-grade ovarian serous cancer. Am J Clin Pathol 2014; 141: 404-414.

[15] Malpica A, Deavers MT, Lu K, et al. Grading ovarian serous carcinoma using a two-tier system. Am J Surg Pathol 2004 28: 496-504.

[16] McDicken IW, McLaughlin PJ, Tromans PM, et al. Detection of placental-type alkaline phosphatase in ovarian cancer. Br J Cancer 1985; 52: 59-64.

[17] Moore RG, MacLaughlan S, Bast RC Jr. Current state of biomarker development for clinical application in epithelial ovarian cancer. Gynecol Oncol 2010; 116: 240-245.

[18] Nakopoulou L, Stefanaki K, Janinis J, et al. Immunohistochemical expression of placental alkaline phosphatase and vimentin in epithelial ovarian neoplasms. Acta Oncol 1995; 34: 511-515.

[19] Rosen DG, Wang L, Atkinson JN, et al. Potential markers that complement expression of CA125 in epithelial ovarian cancer. Gynecol Oncol 2005; 99: 267-277.

[20] Rossi A, Braghin C, Soldano F, et al. A proposal for a new scoring system to evaluate pelvic masses: Pelvic Masses Score (PMS). Eur J Obstet Gynecol Reprod Biol 2011; 157: 84-88.

[21] Saini D, Kala M, Jain V, et al. Targeting the active site of the placental isozyme of alkaline phosphatase by phage-displayed $\mathrm{scFv}$ antibodies selected by a specific uncompetitive inhibitor. 
BMC Biotechnol 2005; 5: 33.

[22] Steffensen KD, Waldstr?m M, Brandslund I, et al. The prognostic and predictive value of combined HE4 and CA-125 in ovarian cancer patients. Int J Gynecol Cancer 2012; 22: 14741482.

[23] Stendahl U, Lindgren A, Tholander B, et al. Expression of placental alkaline phosphatase in epithelial ovarian tumours. Tumour Biol 1989; 10: 126-132.

[24] Stigbrand T, Riklund K, Tholander B, et al. Placental alkaline phosphatase (PLAP)/PLAP-like alkaline phosphatase as tumour marker in relation to CA 125 and TPA for ovarian epithelial tumours. Eur J Gynaecol Oncol 1990; 11: 351-360.

[25] Tholander B, Taube A, Lindgren A, et al. Pretreatment serum levels of CA-125, carcinoembryonic antigen, tissue polypeptide antigen, and placental alkaline phosphatase in patients with ovarian carcinoma: influence of histological type, grade of differentiation, and clinical stage of disease. Gynecol Oncol 1990; 39: 26-33.

[26] Tonik SE, Ortmeyer AE, Shindelman JE, et al. Elevation of serum placental alkaline phosphatase levels in cigarette smokers. Int J Cancer 1983; 31: 51-53.

[27] Trimbos JB, Parmar M, Vergote I, et al. International Collaborative Ovarian Neoplasm trial 1 and Adjuvant ChemoTherapy In Ovarian Neoplasm trial: two parallel randomized phase III trials of adjuvant chemotherapy in patients with early-stage ovarian carcinoma. J Natl Cancer Inst 2003; 95: 105-112.

[28] Wang J, Gao J, Yao H, et al. Diagnostic accuracy of serum HE4, CA125 and ROMA in patients with ovarian cancer: a meta-analysis. Tumour Biol 2014; 35: 6127-6138. 